\title{
Conceptual Design of Four Rotors Flying Car Based on Bionics
}

\author{
Shigang Wang ${ }^{1, a}$, Jian $\mathrm{Li}^{2, \mathrm{~b}}$, Xueshan Gao ${ }^{2, \mathrm{c}}$, B.H. Yan ${ }^{2, a}$ \\ ${ }^{1}$ School of Electrical and Information Engineering, Guangxi University of Science and Technology, \\ Liuzhou, 545006, China \\ ${ }^{2}$ Robotics Institute, Guangxi University of Science and Technology, Liuzhou, 545006, China \\ a1784047367@qq.com, b32557658@qq.com, '916573332@qq.com
}

Keywords: Conceptual Design, Four Rotors, Flying Car, Bionics

\begin{abstract}
With the development of industry and the progress of society, a kind of vehicle that can travel on the ground like a car and fly in the air like an airplane has become a research focus both at home and abroad. The method of bionic design and aerodynamic design method based on the combination of aesthetic principles, through the research on the structure of the four rotor aircraft, the aircraft take-off mode is very suitable for the flying car takeoff: VTOL flight vehicle design inspiration, four rotor flight vehicle with four rotors aircraft taking off; through the method of bionic design and the aerodynamic optimization method, streamlined style. Using 3D software to build a model, a beautiful four rotor flying car is designed to meet the vertical take-off and landing.
\end{abstract}

\section{Introduction}

When the first automobile was born in 1886, the automobile pushed the social progress and became an indispensable part of people's daily life [1] [2]. But in twenty-first century, the short span of a dozen years, the car into a leap forward development, car ownership continues to rise, so urban traffic congestion has occurred. Faced with such enormous traffic pressure, a new type of transportation vehicle, the flying car, will be summoned by the times.

The development history of domestic and foreign flying cars started in the early twentieth century. Even though they didn't have the popularity and popularization like automobiles, many inventors still paid great attention to it. Flying cars can not only travel on the ground like cars, but also fly in the air [3]. As a new concept of transportation, flying cars have formed a research upsurge both at home and abroad [4]. The flying car is a combination of aircraft and automobile body, with aircraft and vehicles, people are attempting to combine the aircraft and cars, designed a flying car, it seems that this idea is a perfectly logical and reasonable, but this idea is a complex process, it is very difficult to achieve [5].

In 1986, was hailed as a pioneer of modern flight vehicle Moore, Taylor, he will be the modern automotive and aerospace technology, developed a can not only run on the ground, and can fly in the air of the car, so that the people of the flying car yearning into reality, and therefore has been issued by the United States government flight license [6]. In 1990s, the design of flying cars was even more optimized. The Terrafugia, designed by the American company transition, solves the problem of Moulton Taylor flying cars because the wing is too wide for everyday use. Terrafugia uses computer technology in a flying car, equipped with an electronic computer, which can be used to adjust the balance when flying in the air [7]. At the same time, the car has aerodynamic buffers, equipped with airbags, and more GPS satellite navigation system. Although transition flying car is a great breakthrough in the flight vehicle design, has solved some problems, but can not solve the car before the flight, because the car needs to take off, will need to start off the wing long distance, more need to glide into power, therefore, transition flying cars still have defects to be ignore [8]. Aeromobil, the Slovakia based company, launched the Aeromobil flying car 3 on the "festival of pioneers" held in Austria in October 29, 2014. The aircraft can complete the dual task of car and aircraft; it can in a short period of time from the car form into aircraft form [9]. The wings of the speed car can be folded and will not take up much width when traveling on the road. This design is a great breakthrough. 


\section{Bionics Design Method}

The shape design of flying vehicle is a design category with high technology content and aesthetic feeling combined with technology. The use of appropriate design methods has important implications for the realization of flying cars. Bionics is the study of a biological or a group of organisms, refining the shape, structure, and other elements of a creature, to design products, or to develop new technologies and scientific disciplines. In the history of industrial design, bionics is applied to many products, transport aircraft and automobile etc. the other is particularly typical, bionics is applied to vehicle design, improve the performance of the vehicles, and even promote the vigorous development of the transportation industry; bionic product design, to design a rigorous theory of bionics shape very beautiful, artistic beauty, which reflects the art and science of bionic design. Then, the design of flying cars should also make full use of bionics, so that the shape of its flying car is more perfect.

Research on method of bionic design is more complex, designers first selected a creature as the research object, through the perception of biological and physiological characteristics, summarized its biological principle, its physiological structure based on the abstract elements. Then establish the concept of bionic design, and then design a variety of sketches, the concept of product design transformation, during which must follow the aesthetic rules, and finally get the ultimate program.

Bionic design is a design method of the system, there is a great help for the design of flight vehicle; use Bionics in the conceptual and shape of the design, let it quickly, there is harmony of the whole scheme; bionic design reflects a extension and sublimation of the basic needs and meaning of general line, it embodies a kind of humanistic care to human nature. For example, the success of the beetle's bionic design illustrates the merit of bionic design in automotive products, and as a combination of cars and airplanes - flying cars should also be designed with biomimetic designs.

\section{The Definition of the Four Rotor Flying Car}

The four wing flying car is a new member of the flying car family, a flying car based on the framework of the four rotor aircraft, which is a brand new product. Its definition comes from the four rotorcraft. Four rotor flying car has the advantages of four rotor aircraft, such as simple handling, unconditional vertical take-off and landing.

Four Rotor Aircraft and Structural Framework. Four rotor aircraft, also known as the four rotor helicopter, is a 4 propeller, and the propeller is square crossing. It has an underactuated system with six degrees of freedom (position and attitude) and 4 control inputs (rotor speed), featuring multivariable, nonlinear, strong coupling, and interference sensitive features, as shown in Figure 1.

The direct power source of four rotor aircraft is composed of four symmetrical around the rotor to provide the same level of the distribution of the four rotor, and the rotor radius are exactly the same, of course, the structure is the same, so the structure for the flight is very necessary, keep the balance in the air. 1 and 3 is rotating counterclockwise, clockwise rotation of rotor 2 and 4, as shown in Figure 2, the middle bracket vehicle space flight control computer and external devices on both ends of the bracket are symmetrically arranged with four motors.

The structural advantage of the four rotor aircraft is that the flying car needs to be used for reference. From the structure of the four rotor aircraft, by rotating the rule four motor with each other in the air, realize six degrees of freedom and the changes in the space of six degrees of freedom can be changed at any time according to need, this is mainly due to the flight control system of aircraft collocation, can have a variety of flight mode according to the different needs of different flight and flight environment for real-time intelligent switching. Four, the rotor can achieve vertical take-off and landing, four motors rotating vigorously, four rotor for vertical take-off and landing to provide sufficient power, without sliding off, in any condition can be achieved take-off and landing. This is also the take-off way the flying car will learn. The main power of the four rotor aircraft is provided by four motors. Before that, it also introduced how to realize the rising, falling, forward and backward flight paths in the space. And there are computer systems that control the various flight modes of an 
aircraft, and it is not difficult to achieve autopilot. Computer systems control flight models of flying cars, and the ability to drive automatically during flight is also a matter of consideration.

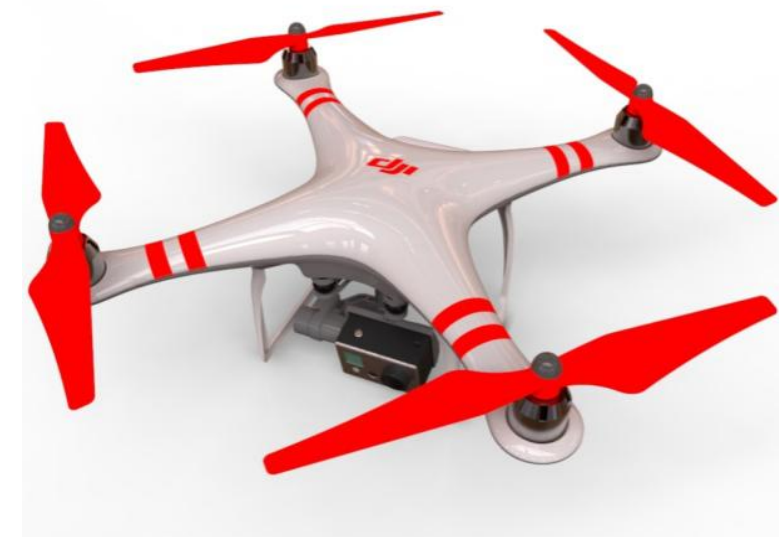

Fig. 1 Four rotor aircraft

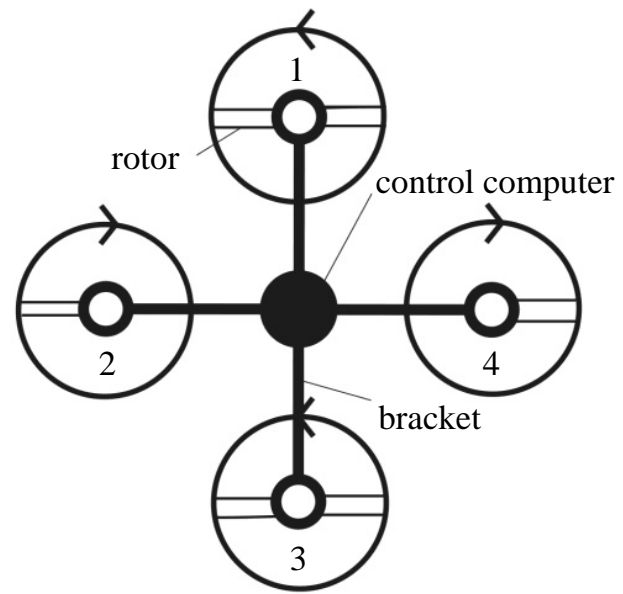

Fig. 2 Structure form

Vertical Take-off and Landing Mode. By introducing the four rotor aircraft, the concept of the four rotor flying vehicle is put forward. The flying car to meet the vertical takeoff can use the rotor structure, then the four rotor aircraft was considered, and the vehicle's wheels and four rotor aircraft wings can be combined with the characteristics of design, this design can also solve the strange appearance of a flying car.

The four rotors have four rotors, and the car has four wheels. The original idea is that the wheels combine with the wings. As shown in Figure 3, in flight mode, the rotor is operated and the wheels are retracted; as shown in Figure 4, the rotor structure is retracted and the wheels are operated when traveling. The flying modes and driving modes of the flying car can be switched at any time.

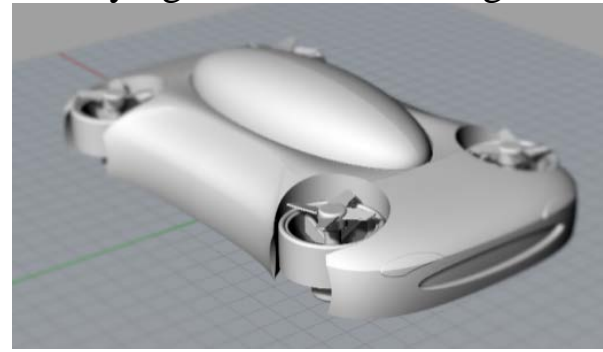

Fig. 3 Flying pattern

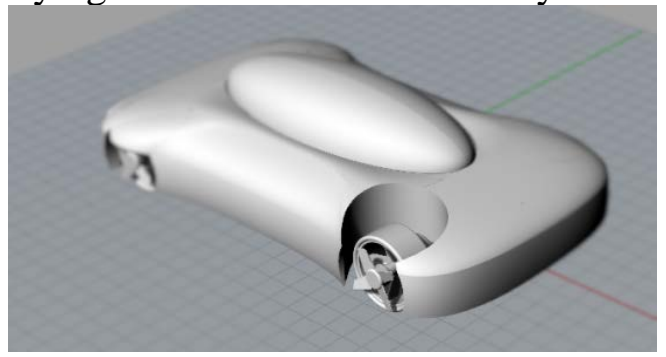

Fig. 4 Driving pattern

Shape Design Process of Four Rotors Flying Car. Four rotor flight vehicle design process, through the study on the structure and properties of the four rotor aircraft design, combined with the car, first based on the bionics design theory, introducing the principle of aesthetics, design a general form, and then introduce the knowledge of aerodynamics of a flying car in aerodynamic shape design, and then combine the aesthetic idea of local details adjust the flight vehicle, draw a sketch, finally using Rino 3D modeling.

Four rotor flight vehicle conceptual design process: a study of a combination of car and four rotor aircraft; bionics theory and aesthetic theory; flying car sketches; using aerodynamic theory; flight vehicle aerodynamic shape; combined with the analysis of the aesthetic theory of local design; sketch; three dimensional modeling; rendering.

\section{Conceptual Design of Four Rotor Flying Car}

The Overall Styling Sketch of a Flying Car. The flying car only in the vertical take-off and landing can not in the restricted conditions, showed up and heroic, so the positioning of the flying car is vtol. VTOL performance jumped in an emergency and the state of the four rotors reminiscent of biological four legged creature, will can't help think of "frog", as shown in Figure 5.

The frog jump way to walk on land, is very consistent with VTOL flying car sprang up; and the frog's four legs can shrink and stretch, and before the definition of the rotor speed retracted and down 
very well; streamlined external contour frog and theoretical aerodynamics can be a very good combination, does not conflict. Bionic design will be carried out with the concept of "leap".
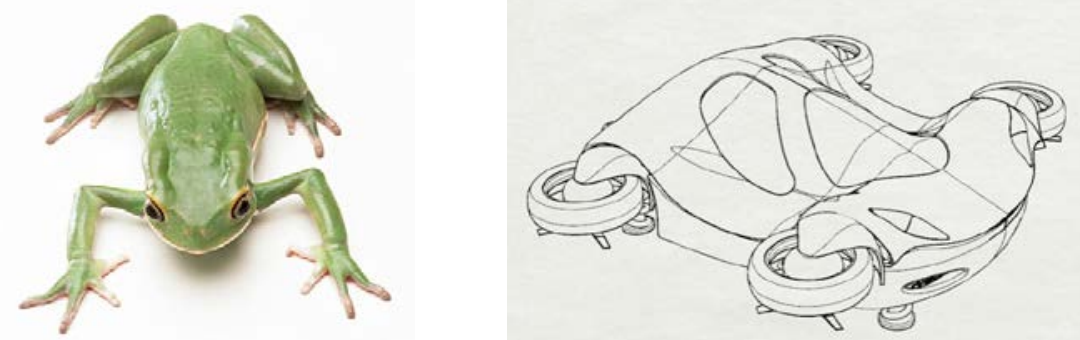

Fig. 5 The frog and the flying car

This flight car sketch, and program a different, mainly to "God" bionic based. A systematic study of the frogs looks, similar to the manner of the frog. The thick lordosis, contour lines had streamlined processing, a linear ratio for the treatment effect is relatively soft and changeable, and the frog head spirit. Larger changes in the width of the body, the length to width ratio of nearly 1 , and the four rotor length ratio of the same, the stability will be better, higher safety, of course, aerodynamic shape will be reduced, this is not the main factor.

The Combination of Aerodynamic Optimization Design and Aesthetics Design. Air resistance can be obtained by experiment model in the scheme is relatively small, but this model is not a final conclusion, the initial design of the model does not consider the rearview mirror and other accessories, car and other details of the aerodynamics and aesthetic principle factors. In order to get the perfect design scheme, aerodynamic optimization design of the front face, front and rear lights, rotor structure and cab of the flying car will be carried out, and a successful scheme combined with the aesthetic principle should be designed.

The whole body contour is inspired by the frog's body lines. After studying the shape of the frog, it refines the more refined shape and makes proper exaggeration and modification. The graceful back curve of the car is perfectly integrated with the contour curve of the cab, and the three cambered glass windows fit perfectly with the cab shape, thus interpreting the perfect combination of aerodynamics and aesthetics.

Conceptual Design of Front Face of Flying Car. Front face part has all sorts of lamps and lanterns, bumper, trademark and so on. Since the flying car will use electric power output, most of the front face will not be occupied by the intake grille; most of the front face will be the whole surface. Arrangement to coordinate the relationship between the transition relations in the design shall be handled carefully and properly and the surface shape of each component should pay attention to the virtual and real change, loose and dense: General in a large blank layout an exquisite trademarks, so as to make the finishing point.

As shown in Figure 6, the front bumper section has a convex shape, and the transition part is smooth, so that the airflow can be smoothly split and turned to the back, so that the airflow can not be blocked. An integral structure is formed between the position of the front bumper and the body of the vehicle body, and the gap and the resistance and the lift force are greatly reduced.

Front and Rear Headlight Design. Headlight design: headlights are like eyes on the human face, as shown in Figure 7. Headlight design inspired by the fox's eyes, the fox has a pair of spiritual and sharp eyes, and in order to increase the agility of the car, you can design and fox eyes like headlights modeling. The headlights through exaggerated processing makes the contour curve radian increases, appear in the whole body smooth and clean face, sharp eyes full of spirit, and in contrast to contrast with the front chin thick car string.

Structure Design of Flying Vehicle Rotor. As shown in Figure 8, the design of the rotor structure in a flying automobile combines the wheel structure with the rotor structure, and the power of the wheel and rotor comes from the same motor. In flight mode, put down the support frame, hold the flying car; turn the wheel up 90 degrees, and the ground level, the rotor open, rotating can work. When the land is moving, the wheels are lowered, the rotor fold, hidden inside the wheel, the support frame retracted, and the wheels can work. The rotor can be folded to increase the length of the rotor 
and increase the lift at which the rotor works. The wheel rotation mechanism is a peculiar shape, with streamlined style spherical cover, consistent with the overall speed of the streamlined appearance, and plays a role of connection, rotating the wheel horizontal or vertical spherical cover, thereby changing flight and driving mode.

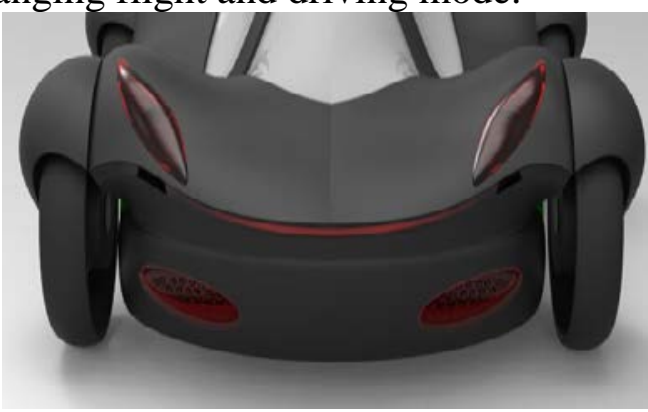

Fig. 6 Design of the front face

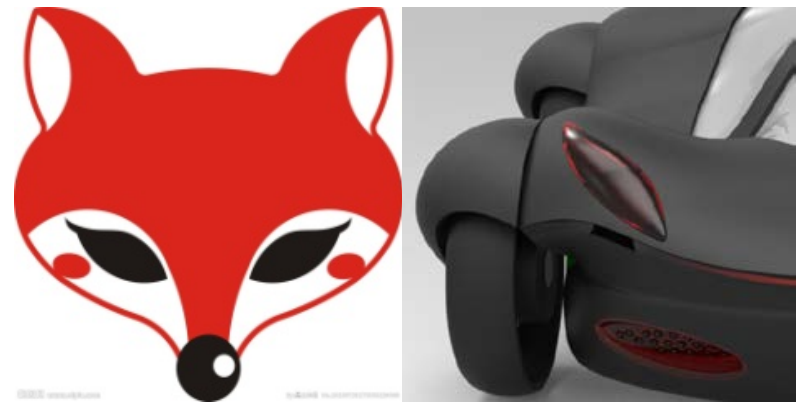

Fig. 7 Headlamps design

\section{Three Dimensional Shape Model of Flying Car}

The modeling should ensure that the model is exactly the same as the shape of the real object. Secondly, the subdivision of the model surface should be taken into account because it directly affects the editing and coloring effects of the model. According to the scheme chosen by the sketch, the model is designed by using Rhino modeling software and combining the relevant contents of aerodynamics.
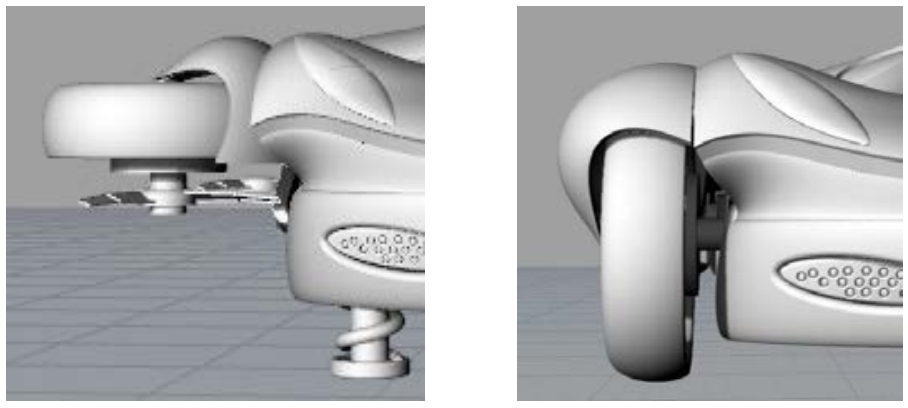

Fig. 8 The rotor structure

As shown in Figure 9, the design of the vehicle with the "frog" form and manner as design elements, through the design and optimization of the skillful combination of aesthetic theory, both the flight mode and vehicle mode, highlighting the unique image of the flying car.
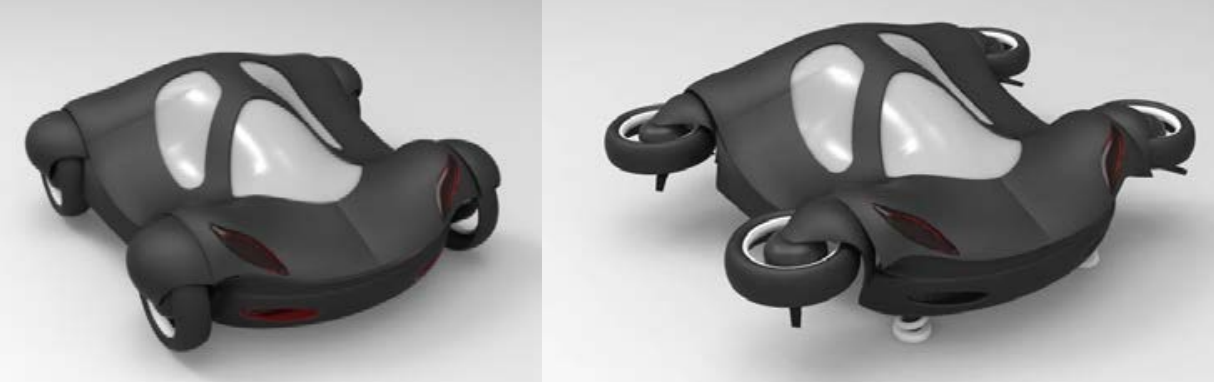

Fig. 9 Three dimensional shape model of flying car

The front of the car sinks, the tail rises, showing its aggressiveness, there is a frog jumped up, ready to take the impulse; the front to frog shape as inspiration, through refining its appearance elements, forward tilt, tilt angle prominent. The mask is perfectly connected with the front face and the bumper; the body shape surface is integrated with the driver's cab, and the door is hidden without a prominent edge. Other fox eye headlights, very prominent, to speed to spiritual feeling; light bumper, shape is simple, but elegant, this is to highlight the unique charm of the headlights taillights; delicate and spirituality, taillight shape design of semi open, the bottom of the lamp to highlight the tail of the charming and moving; after the bumper and the vehicle body are convergence, smooth transition, rear bumper looks more powerful, dynamic; roof cab design, three huge curved glass 
combination, like a giant mask, and body surface integrated, improve the dynamic performance of the whole air.

\section{Conclusion}

This paper mainly designs the shape of the four rotor flying vehicle, and explores two problems to be solved. One is to design a flying vehicle suitable for vertical take-off and landing, and two, to make the flying car beautiful in appearance.

Through the study of the structure of the four rotor aircraft, it is found that the take-off mode of the four rotor aircraft is very suitable for the vertical take-off and landing of the flying vehicle. Therefore, the flying vehicle is designed for the inspiration of the four rotor take-off. The bionic design method and aerodynamic method are adopted to optimize the design style. A three dimensional model is built to design a four rotor flying vehicle with beautiful appearance and in situ flight.

The research shows that the design of the flying car, but there are lots of beautiful and achieve vertical take-off and landing are few, and this four rotor flight vehicle design, ingenious combination of rotor and wheel, the rotor mechanism of hiding and VTOL, both to meet the aesthetic and functional realization. It's a flying car worth studying. It needs more people to study and design it.

\section{Acknowledgement}

This work was supported by Liuzhou City Science Research and Technology Development Project (Grant No. 2016C050203) and Doctoral Foundation of Guangxi University of Science and Technology (Grant No. XKB17Z08).

\section{References}

[1] CAO Feng, YAO Mingtao, LEI Xueyuan. Present situation and prospects of flying cars. Modern Machinery, (2), 2015, pp. 89-93.

[2] Lichen Zhang. Analysis of conceptual flying car design. Beauty \& Times, (5), 2016, pp. 113-115.

[3] Kuktae Kim, Kyoil Hwang and Hoonmo Kim. Study of an adaptive fuzzy algorithm to control a rectangular-shaped unmanned surveillance flying car. Journal of Mechanical Science and Technology, 27, 2013, pp. 2477-2486.

[4] Godfrey Derek Sams. Conceptual design and analysis of ferrari F430 flying car. International Journal of Research in Engineering and Technology, 1, 2012, pp. 303-306.

[5] B Saeed and G. B. Gratton. An evaluation of the historical issues associated with achieving non-helicopter V/STOL capability and the search for the flying car. Aero, 114, 2010, pp. 91-102.

[6] Lim J W, Chopra I. Aeroelastic optimization of helicopter rotor using an efficient Sensitivity analysis. Journal of Aircraft, 28, 1991, pp. 123-129.

[7] Taylor R B. Helicopter vibration reduction by rotor blade modal shaping. In: Proceedings of the 38th Annual Forum. Maryland: American Helicopter Society, 1982, pp. 223-229.

[8] SU Jing-ya, ZHANG Rui-fen, WANG Xin-hua, CAI Kai-yuan. Controlling a four-rotor aircraft based on noise-attenuation differentiator. Control Theory \& Applications, 26, 2009, pp. 827-828.

[9] Su Jingya, Fan Penhui, Cai Kaiyuan. Attitude control of quad rotor aircraftvia nonlinear PID. Journal of Beijing University of Aeronautics and Astronautics, 37, 2011, pp. 1054-1055. 